\title{
Note on a formula connected with Fourier Series.
}

\author{
By Professor H. M. Macdonald. \\ (Received 12th December 1925. Read 2nd May 1925.)
}

An expression for the sum of a series of terms which takes account of the different rates of oscillation of the functions involved is of importance for series such as occur in the problems of diffraction, and a relation of this kind has been given by Poisson.* This relation can be written in the form

$$
S_{m}=\frac{1}{2}\left(u_{n}-u_{n+m}\right)+\int_{0}^{m} u_{n+t} d t+2 \underset{k=1}{k=\infty} \int_{0}^{m} u_{n+t} \cos 2 k \pi t d t,
$$

where $S_{n}$ denotes the sum of $m$ terms of the series $\Sigma u_{n}$ beginning with the term $u_{n}$. This formula can be deduced from Dirichlet's integral, for

$$
\begin{aligned}
\int_{a}^{b} \frac{\sin (2 k+1) \pi t}{\sin \pi t} f(t) d t \rightarrow \frac{1}{2}[ & f\left(n_{1}-\right)+f\left(n_{1}+\right) \\
& \left.+f\left(n_{1}+1-\right)+\ldots+f\left(n_{2}+\right)\right],
\end{aligned}
$$

when $k \rightarrow \infty$, where $n_{1}, n_{2}$ are the two integers which satisfy the relations

$$
n_{\mathrm{i}}>a>n_{1}-1, n_{2}+1>b>n_{2},
$$

and therefore

$$
\begin{aligned}
\frac{1}{2}\left[f\left(n_{1}-\right)+f\left(n_{1}+\right)+\ldots\right. & \left.+f\left(n_{2}+\right)\right] \\
& =\int_{a}^{b} f(t) d t+2 \int_{a}^{b} \sum_{k=1}^{k=\infty} f(t) \cos 2 k \pi t d t,
\end{aligned}
$$

where $f(t)$ is a function of $t$ satisfying the Dirichlet conditions. It follows that

$$
\begin{aligned}
\frac{1}{2}\left[f\left(n_{1}-\right)+\right. & \left.f\left(n_{1}+\right)+\ldots+f\left(n_{2}+\right)\right] \\
& =\int_{a}^{b} f(t) d t+2 \sum_{k=1}^{k=\infty} \int^{b} f(t) \cos 2 k \pi t d t
\end{aligned}
$$

\footnotetext{
* Yem. de l'Acad. des Sciences, t, VI, pp. 5-78, 5-91,
} 
when the series on the right hand side converges, or

$$
\sum_{n=n_{1}}^{n=n_{2}} u_{n} \int_{a}^{b} u_{t} d t+2 \sum_{k=1}^{k=\infty} \int_{a}^{b} u_{t} \cos 2 k \pi t d t
$$

when $a$ is an integer the first term on the left hand side of relation (1) has to be omitted, and when $b$ is an integer the last term on the left hand side of relation (1) has to be omitted; therefore

$$
\begin{aligned}
& \underset{\substack{n=n_{1} \\
n=n_{n}}}{n=\frac{1}{2} u_{n}} u_{n_{1}}+\int_{1}^{b} u_{t} d t+2 \sum_{k=1}^{k=\infty} \int_{n_{1}}^{b} u_{t} \cos 2 k \pi t d t \\
& \underset{n=n_{1}}{n=n_{2}}=\frac{1}{2} u_{n_{2}}++\int_{a}^{n_{2}} u_{t} d t+2 \sum_{k=1}^{k=\infty} \int_{a}^{n_{2}} u_{t} \cos 2 k \pi t d t \\
& \sum_{n=n_{1}}^{n=n_{2}} u_{n}=\frac{1}{2}\left(u_{n_{1}-}+u_{n_{2}+}\right) \\
& +\int_{n_{1}}^{n_{2}} u_{t} d t+2 \sum_{k=1}^{k=\infty} \int_{n_{1}}^{n_{2}} u_{t} \cos 2 k \pi t d t, \\
& \sum_{n=n_{1}}^{n=n_{n}} u_{n}=\frac{1}{2}\left(u_{n_{1}-}-u_{n_{2}+1-1-}\right) \\
& +\int_{n_{1}}^{n_{2}+1} u_{t} d t+2 \sum_{k=1}^{k=\infty} \int_{n_{1}}^{n_{2}+1} u_{t} \cos 2 k \pi t d t
\end{aligned}
$$

or

which is the same as the relation

$$
\begin{aligned}
S_{m}=\frac{1}{2}\left(u_{n}-u_{n+m}\right)+\int_{0}^{m} u_{n+\imath} d t & \\
& +2 \sum_{k=1}^{k=\infty} \int_{0}^{m} u_{n+t} \cos 2 k \pi t d t
\end{aligned}
$$

when $S_{m}$ is the sum of $m$ terms of the series beginning with the term $u_{n}$.

When $u_{x}$ oscillates slowly as $x$ varies, the first integral on the right hand side is more important than any of the others; the formula obtained for the approximate value of the sum by neglecting all the subsequent integrals is effectively the same as that given by Maclaurin.*

If, however, the rate of oscillation of $u_{x}$ is in the neighbourhood of the rate of oscillation of $\cos 2 p \pi x$, where $p$ is an integer, the

\footnotetext{
- Treatise on Fluxions, Bk. II, Ch. IV.
} 
most important term on the right hand side is the integral $\int_{0}^{m} u_{n+t} \cos 2 p \pi t d t$, and, if the rate of oscillation of $u_{x}$ is in the neighbourhood of the rate of oscillation of $\cos (2 p+1) \pi x$, the consecutive integrals on the right hand side $\int_{0}^{m} u_{n+t} \cos 2 p \pi t d t$ and $\int_{0}^{m} u_{n+t} \cos 2(p+1) \pi t d t$ are of equal importance and contribute the most important part of the sum. Again, it may happen that there is a group or groups of terms of a series whose sum constitutes the most important part of the sum of the series. In this case, if the terms of one of the groups lie between $u_{n_{1}}$ and $u_{n_{2}}$, the sum of the terms of this group is by the preceding

$$
\int_{a}^{b} u_{t} d t+2 \sum_{k=1}^{k=\infty} \int_{a}^{b} u_{t} \cos 2 k \pi t d t ;
$$

and, in general, the origin of $t$ being chosen conveniently, it will be possible to write from the range from $a$ to $b$

where

$$
u_{t}=v_{t}+w_{t},
$$

$$
\int_{-\infty}^{\infty} v_{t} \cos 2 k \pi t d t-\int_{a}^{b} v_{t} \cos 2 k \pi t d t+\int_{a}^{b} w_{t} \cos 2 k \pi t d t
$$

can be neglected for all values of $k$, and the principal part of the sum due to this group is then

$$
\int_{-\infty}^{\infty} v_{l} d t+2 \sum_{k=1}^{k=\infty} \int_{-\infty}^{\infty} v_{t} \cos 2 k \pi t d t
$$

the most important term in this expression depending on the rate of oscillation of $v_{t}$. An example of this is afforded by the series that occur in connection with the effect of a spherical obstacle on a train of waves.

The formula can also be used to obtain certain analytical relations, and some examples of this are given.

(a) Writing in the relation $\left(5^{\prime}\right) u_{n}=n^{2}$, the result is

$$
\sum_{n=1}^{n=m} n^{2}=\frac{1}{2}\left\{1-(m+1)^{2}\right\}+\int_{0}^{m}(t+1)^{2} d t+2 \sum_{k=1}^{k=\infty} \int_{0}^{m}(t+1)^{2} \cos 2 k \pi t d t,
$$


whence, integrating the terms on the right hand side by parts, $\frac{1}{6} m(m+1)(2 m+1)+\frac{1}{2}\left\{(m+1)^{2}-1\right\}-\frac{1}{3}\left\{(m+1)^{3}-1\right\}=m \sum_{k=1}^{k=\infty} \frac{1}{k^{2} \pi^{2}}$, that is $\quad \frac{1}{6}=\sum_{k=1}^{k=\infty} \frac{1}{k^{2} \pi^{2}}$ or $\sum_{k=1}^{k=\infty} \frac{1}{k^{2}}=\frac{\pi^{2}}{6}$.

(b) Writing $u_{n}=\cos n z$, the result is

$$
\begin{aligned}
& \quad \sum_{r=0}^{r=m} \cos (n+r) z=\frac{1}{2}\{\cos n z-\cos (n+m) z\} \\
& \quad+\int_{0}^{m} \cos (n+t) z d t+2 \sum_{k=1}^{k=\infty} \int_{0}^{m} \cos (n+t) z \cos 2 k \pi t d t,
\end{aligned}
$$

that is

$$
\begin{aligned}
& \left\{\sin \left(n+m-\frac{1}{2}\right) z-\sin \left(n-\frac{1}{2}\right) z\right\} / 2 \sin \frac{1}{2} z=\frac{1}{2}\{\cos n z-\cos (n+m) z\} \\
& \quad+\{\sin (n+m) z-\sin n z\}\left\{\frac{1}{z}+\sum_{k=1}^{k=\infty}\left(\frac{1}{z+2 k \pi}+\frac{1}{z-2 k \cdot \pi}\right)\right\},
\end{aligned}
$$

whence

$$
\begin{aligned}
& \frac{1}{2}\{\sin (n+m) z-\sin n z\} \cot \frac{1}{2} z \\
& \quad=\left\{\sin (n+m) z-\sin n z ;\{\frac{1}{z}+\underbrace{k=\infty}_{k=1}\left(\frac{1}{z+2 k \pi}+\frac{1}{z-2 k \pi}\right)\},\right.
\end{aligned}
$$

that is

$$
\frac{1}{2} \cot \frac{1}{2} z=\frac{1}{z}+\sum_{k=1}^{k=\infty}\left(\frac{1}{z+2 k \pi}+\frac{1}{z-2 k \pi}\right) .
$$

(c) If relation $\left(\tilde{b}^{\prime}\right)$ is applied to the terms on the right hand side of the identity

$$
\sinh \eta / \cosh \eta-\cos \xi)=1+2 \sum_{n=1}^{n=\infty} e^{-n \eta} \cos n \xi
$$

the result is

$$
\begin{aligned}
\sinh \eta /(\cosh \eta-\cos \xi)=1-1+2 & \int_{0}^{\infty} e^{-t \eta} \cos \xi t d t \\
& +4 \sum_{k=1}^{k=\infty} \int_{0}^{\infty} e^{-t \eta} \cos \xi t \cos 2 k \pi t d t,
\end{aligned}
$$

that is

$\sinh \eta /(\cosh \eta-\cos \xi)=2 \eta /\left(\eta^{2}+\xi^{2}\right)$

$$
+\sum_{k=1}^{k=\infty}\left[2 \eta /\left\{\eta^{2}+(\xi+2 k \pi)^{2}\right\}+2 \eta /\left\{\eta^{2}+(\xi-2 k \pi)^{n}\right\}\right] .
$$


(d) The relation

can be written

$$
S=1 / a+2 \sum_{m=1}^{m=\infty} a \cos n z /\left(a^{2}+n^{2}\right)
$$

$$
S=\sum_{n=-\infty}^{n=\infty} a \exp (i n z) /\left(a^{2}+n^{2}\right),
$$

and applying the formula this becomes

$$
S=\int_{-\infty}^{\infty} a e^{i z t} /\left(a^{2}+t^{2}\right) d t+2 \sum_{k=1}^{k=\infty} \int_{-\infty}^{\infty} e^{i z t} \cos 2 k \pi t /\left(a^{2}+t^{2}\right) d t,
$$

that is, when $2 \pi>z>0$,

$$
S=\pi e^{-a z}+\sum_{k=1}^{k=\infty} \pi e^{-a(\xi+2 k \pi)}+\sum_{k=1}^{k=\infty} \pi e^{-a(2 k \pi-z)},
$$

or $S=\pi e^{-a z} /\left(1-e^{-2 a \pi}\right)+\pi e^{-a(2 ; \pi-z)} /\left(1-e^{-2 a x}\right)$

when $0>\approx>-2 \pi$,

$$
=\pi \cosh a(\pi-z) / \sinh a \pi
$$

$$
S=\pi e^{u z}+\sum_{k=1}^{k=\infty} \pi e^{-a(z+2 k \pi)}+\sum_{k=1}^{k=\infty} e^{-a(2 k x-i)}
$$

or $S=\pi \cosh a(\pi+z) / \sinh a \pi$.

(e) Applying the formula to the expression

$$
f(a, b)=\sum_{n=-\infty}^{n=\infty} e^{-a n^{2}-2 \iota n},
$$

where the real part of $a$ is positive,

$f(a, b)=\int_{-\infty}^{\infty} e^{-a t "-2 b t} d t+2 \sum_{l=1}^{k=\infty} \int_{-\infty}^{\infty} e^{-a t=-2 b t} \cos 2 k \pi t d t$,

whence

$$
\begin{aligned}
f(a, b)=(\pi / a)^{\frac{1}{2}}\left[\exp \left(b^{2} / a\right)+\right. & \stackrel{\sum_{k=1}^{k=\infty} \exp \left\{\left(b^{2}-k^{2} \pi^{2}\right) / a-2 k b \pi i / a\right\}}{ } \\
& +\sum_{k=1}^{k=\infty} \exp \left\{\left(b^{2}-k^{2} \pi^{2}\right) / a+2 k b \pi i / a_{j}\right\}
\end{aligned}
$$

or

$f^{\prime}(a, b)=(\pi / a)^{\frac{1}{2}} e^{b^{2}} / u \sum_{k=-\infty}^{k=} \exp \left(-k^{2} \pi^{2} / a-2 k b \pi i / a\right) ;$

that is, writing

the relation is

$$
a_{1}=\pi^{2} / a, b_{1}=\pi b i / a,
$$

$$
\text { at } e^{-b y / 2 a} f(a, b)=a_{1}^{t} e^{-b z_{1} / 2 a_{1}} f^{\prime}\left(a_{1}, b_{1}\right) \text {. }
$$


The formula can also be applied to a single term, for example.

$$
\cos m z=\int_{m-t}^{m+t^{\prime}} \cos z t d t+2 \sum_{k=1}^{k=\infty} \int_{m-t}^{m+t^{\prime}} \cos z t \cos 2 k \pi t d t,
$$

where $1>t>0,1>t^{\prime}>0$, and $m$ is a positive integer, whence $\cos m z=\cos m z\left[\left(\sin t^{\prime} z+\sin t z\right) / z\right.$

$$
\begin{aligned}
& +\sum_{k=1}^{k=\infty}\left\{\sin t^{\prime}(z+2 k \pi)+\sin t(z+2 k \pi)\right\} /(2 k \pi+2) \\
& \left.+\sum_{k=1}^{k=\infty}\left\{\sin t^{\prime}(z-2 k \pi)+\sin t(z-2 k \pi)\right\} /(z-2 k \pi)\right] \\
& +\sin m z\left[\left(\cos t^{\prime} z-\cos t z\right) / z\right. \\
& +\sum_{k=1}^{k=\infty}\left\{\cos t^{\prime}(z+2 k \pi)-\cos t(z+2 k \pi)\right\} /(z+2 k \pi) \\
& \left.+\sum_{k=1}^{k=\infty}\left\{\cos t^{\prime}(z-2 k \pi)-\cos t(z-2 k \pi)\right\} /(z-2 k \pi)\right],
\end{aligned}
$$

writing $t^{\prime}=t$, this relation becomes

$$
\begin{gathered}
1=2 \sin t z / z+2 \sum_{k=1}^{k=\infty} \sin t(z+2 k \pi) /(z+2 k \pi) \\
\quad+2 \sum_{k=1}^{k=\infty} \sin t(z-2 k \pi) /(z-2 k \pi), \\
\text { or } \sum_{k=-\infty}^{k=\infty} \sin t(z+2 k \pi) /(z+2 k \pi)=\frac{1}{2}, \text { when } 1>t>0 .
\end{gathered}
$$

Substituting this result above, it follows that

$$
\sum_{k=-\infty}^{k=\infty} \cos t(z+2 k \pi) /(z+2 k \pi)=\sum_{k=-\infty}^{k=\infty} \cos t^{\prime}(z+2 k \pi) /(z+2 k \pi),
$$

whence, writing $t^{\prime}=\frac{1}{2}$,

$$
\begin{aligned}
& \stackrel{\sum}{k=\infty}_{k=-\infty}^{k} \cos t(z+2 k \pi) /(z+2 k \pi) \\
& =\cos \frac{1}{2} z \sum_{k=-\infty}^{k=-\infty} \cos k \pi /(z+2 k \pi)=\frac{1}{2} \cot \frac{1}{2} z .
\end{aligned}
$$

Therefore combining these results,

$$
\begin{aligned}
& \stackrel{\substack{k \\
k=-\infty}}{\unrhd} \cos 2 k \pi t /(z+2 k \pi)=\frac{1}{2} \cos \left(\frac{1}{2}-t\right) z / \sin \frac{1}{2} z, \\
& \stackrel{\substack{k=\infty \\
k=-\infty}}{\doteq} \sin 2 k \pi t /(z+2 k \pi)=\frac{1}{2} \sin \left(\frac{1}{2}-t\right) z / \sin \frac{1}{2} z \text {. }
\end{aligned}
$$

